\title{
Kernos
}

Revue internationale et pluridisciplinaire de religion grecque antique

18 | 2005

Varia

\section{La terre-mère : une lecture par le genre et la rhétorique patriotique}

\section{Violaine Sebillotte Cuchet}

\section{(2) OpenEdition \\ Journals}

Édition électronique

URL : http://journals.openedition.org/kernos/1526

DOI : 10.4000/kernos.1526

ISSN : 2034-7871

Éditeur

Centre international d'étude de la religion grecque antique

Édition imprimée

Date de publication : 1 janvier 2005

Pagination : 203-218

ISSN : 0776-3824

Référence électronique

Violaine Sebillotte Cuchet, "La terre-mère : une lecture par le genre et la rhétorique patriotique », Kernos [En ligne], 18 | 2005, mis en ligne le 28 juin 2011, consulté le 23 avril 2019. URL : http:// journals.openedition.org/kernos/1526; DOI : 10.4000/kernos.1526 


\title{
La terre-mère : une lecture par le genre et la rhétorique patriotique
}

\begin{abstract}
Résumé : Cet article discute le thème de l'autochtonie - essentiellement athénienne - en l'intégrant dans la logique du discours patriotique qui est la sienne. La prise en compte des exigences de ce type de discours tout à fait particulier, de même que la prise en compte du renouvellement du regard sur les identités sexuées qu'ont permis les études " genre », permettent aujourd'hui de proposer une interprétation différente de celle de Nicole Loraux. Celle-ci comprenait, dans sa lecture du Ménexène de Platon, le mythe de l'autochtonie comme un «transfert» des compétences reproductives féminines sur un espace tenu par les hommes seuls, façon pour eux de déposséder les femmes de leur "puissance » et de penser la reproduction sans elles. Après avoir montré que les Grecs pensaient généralement la procréation comme une opération mixte et souvent équitablement partagée, surtout dans la tradition littéraire qui précède Aristote, je développe l'hypothèse selon laquelle l'usage du motif de la terre mère est une façon de construire un attachement contraignant (car pensé comme un lien biologique individuel) au territoire civique. Il ne procéderait donc pas d'une lutte des sexes et d'une peur des hommes envers la puissance des femmes, mais serait l'instrument politique qui, à l'intérieur d'une société patrilinéaire où règne la «domination masculine », permet de susciter une relation particulièrement étroite avec le territoire habité.
\end{abstract}

Abstract: The earth mother: a reading through gender and patriotic rhetoric. This article tackles the issue of autochtony - mainly Athenian autochtony - fitting it more than has been traditionally the case into the logic of patriotic discourse from which it emerges. Taking into account the demands of this type of very particular discourse, together with the new perspectives on sexed identities made possible by gender studies, allows us today to offer an interpretation that is different from Nicole Loraux's examining Plato, Menexenus. She understood the myth of autochtony as a "transference" of female reproductive competences to the civic land, an exclusive possession of men and a way for them to dispossess women of their power and to consider reproduction without women. After demonstrating that for the Greeks, mainly in the preAristotelician tradition, males and females were usually equally concerned with the operation of procreation, I will suggest that the use of the mother earth theme is a way of constructing a constraining bond (considered both as a biological and an individual one) between citizens and their civic territory. This bond does not arise from the sex struggle or even from male fear of female power but, in the context of a patrilineal society dominated by males, it is the political instrument for the alienation of citizens from the land they inhabit.

Les récits ou les allusions à des héros ou des hommes nés du sol peuvent être rassemblés dans un seul motif mythique : celui de la maternité de la terre. On reconnaitra que l'essentiel de ce motif est supporté par une métaphore obéissant à la logique du vraisemblable qu'Aristote reconnaissait comme caractéristique du mythe ${ }^{1}$ : elle fonctionne tant qu'il y aura des hommes pour penser que la terre produit des fruits comme une mère semble produire des enfants. Sur cette analogie entre la terre et la mère bien des choses ont été

1 Aristote, Poétique $1447 \mathrm{a}$ et $1450 \mathrm{a}$. 
écrites et bien des controverses restent ouvertes. ${ }^{2}$. Car en ce domaine la perspective a été passablement obscurcie par le fantasme d'une terre qui exprimerait la puissance d'une Grande Déesse, Déesse Terre ou Déesse Mère, à l'origine de tout et en général victime d'un ordre patriarcal magnifié ou détesté qui aurait étouffé sa présence ${ }^{3}$. Elle l'est désormais par l'opprobre qui pèse sur une thématique parfois devenue d'emblée suspecte. Un deuxième obstacle, moins frontal, m'apparaît aujourd'hui : il tient dans la séduisante hypothèse de Nicole Loraux sur l'autochtonie athénienne. Pour rendre compte du mythe de la naissance des citoyens de la terre même de leur cité, mythe participant à l'élaboration de l'idéologie de la cité pensée unifiée dans un présent éternel, elle propose une interprétation enchâssée dans une évidente lutte des sexes : les citoyens d'Athènes, assouvissant leur profond désir d'un monde purement masculin, auraient ainsi trouvé le moyen de se débarrasser symboliquement d'un pouvoir des femmes auquel leur existence était malheureusement contrainte. Quand l'hypothèse se transforme en postulat, ce qui est parfois le cas aujourd'hui, elle devient un nouvel obstacle intellectuel ${ }^{4}$.

Acceptant toutes les conclusions des démonstrations si claires de Stella Georgoudi à propos du caractère très instrumentalisé du pseudo-matriarcat antique, de la richesse et de la diversité des figures de la terre et de la mère, je n'aurai pas de scrupules à parler parfois de «maternité de la terre » car ce singulier ne renvoie, en fin de compte, qu'à une fonction, non à une divinité spécifique. En ce qui concerne le rêve misogyne des Grecs peut-être suffiraitil d'opposer les compagnons et maris athéniens des héroïnes d'Aristophane à l'Hippolyte d'Euripide pour rappeler que, si la sexualité (toujours susceptible d'être procréative) pouvait sembler un bien grand mal, elle était, pour d'autres, source de tels plaisirs qu'ils auraient eu bien du mal à s'en passer !

En partant de l'étude des documents qui évoquent le motif de la maternité de la terre, et non de telle ou telle hypothèse, on peut, me semble-t-il, ouvrir le champ interprétatif et proposer à l'analogie terre-mère une explication qui ne ressortisse plus de la guerre des sexes, en tout cas en première analyse, mais d'une fonction sociale autrement plus précise. Dans cette perspective la

\footnotetext{
2 N. LORAux, "Qu'est-ce qu'une déesse?», in P. SCHMitT PANTEL, Histoire des femmes en Occident, I. L'Antiquité, Paris, 1990, p. 39-79 et particulièrement p. 59-70; S. GEORGOUDI, "Bachofen, la matriarcat et le monde antique. Réflexion sur la création d'un mythe », ibid., p.585602; ead. "Autour d'une anthropologie des sexes », Mètis 9-10 (1994-1995), atelier coordonné par S. Georgoudi, p. 285-334; ead., «Gaia/Gê. Entre mythe, culte et idéologie » in S. DES BOUVRIE (dir.), Myth and Symbol I, Bergen, 2002, p. 113-134. Voir également B. WAGNER-Hasel (éd.), Matriarchatstheorien der Altertumswissenschaft, Darmstadt, 1992 (Wege der Forschung, 651).

3 Aux indications de la note précédente, ajouter P. Borgeaud, La Mère des Dieux, Paris, 1996, qu'il faut opposer à la thèse délirante de F. GANGE, Les Dieux menteurs. Notre mémoire ensevelie : l'bumanité aux temps de la Déesse, Paris, 1998.

4 Des critiques cependant: J. BLOK, "Recht und Ritus der Polis. Zu Bürgerstatus und Geschlechterverhältnissen im klassischen Athen », Historische Zeitschrift, Heft 278/1 (2004), p. 1-16, qui réfléchit sur une définition moins institutionnelle et plus cultuelle de la citoyenneté, définition qui fait une large part aux femmes et relativise le clivage des sexes.

5 Aristophane, Lysistrata, 764; Euripide, Hippolyte, 616-624.
} 
notion de genre m'a semblé être un opérateur intellectuel particulièrement pertinent pour reprendre le dossier. En mettant en question les catégories du masculin et du féminin, elle oblige à préciser davantage encore la nature du corpus. C'est essentiellement un corpus athénien, très politique, des $\mathrm{V}^{\mathrm{e}}$ et surtout $\mathrm{IV}^{\mathrm{e}}$ siècle avant notre ère, qui relève de ce que je désigne comme de la rhétorique patriotique. La nature de ce type de discours oriente d'une toute autre façon l'interprétation du motif de la terre mère.

\section{La nouvelle problématisation du dossier, liée aux études de genre}

Une certaine critique féministe, et je pense particulièrement à la thèse défendue par Françoise Héritier en 1996 puis reprise ensuite, quoique largement modifiée en $2002^{6}$, interprète le dossier de la maternité de la terre, à la suite de Nicole Loraux, dans une perspective d'élucidation des mécanismes de la domination masculine.

Dans ces livres, que je ne saurais examiner d'un point de vue philosophique ou anthropologique, Françoise Héritier explique le phénomène de la domination masculine par le déséquilibre qu'elle observe dans le processus de la reproduction sexuée. Les femmes auraient un rôle extrêmement important dans ce processus et les hommes voudraient les en dessaisir. C'est ainsi qu'interviendraient les mythes autour de la maternité de la terre ${ }^{7}$. Le postulat central est donc celui d'un rapport de force entre les hommes et les femmes autour de la question de la reproduction, avec l'hypothèse corrélée que les hommes ont peur de la «puissance féminine " manifestée dans sa puissance maternelle ${ }^{8}$. Françoise Héritier a formulé plus précisément la hantise des hommes : ce qui leur est intolérable est la «capacité exorbitante» des femmes à produire des enfants des deux sexes, affirme-t-ellè. À partir d'une observation indubitable, la grossesse, seul "privilège» des femmes, l'anthropologue propose une affirmation beaucoup plus contestable selon laquelle les hommes ont toujours pensé que les femmes fabriquaient seules (leur fameux pouvoir exorbitant) les enfants, garçons et filles, accordée à une autre affirmation, que je considèrerai plutôt comme une hypothèse de travail, celle d'un ressentiment unanime des hommes à leur égard.

Cette thèse doit particulièrement intéresser les antiquistes qui, en tant que spécialistes de discours mythiques, sont sollicités. Et si je cite Françoise

\footnotetext{
${ }^{6}$ F. HÉRITIER, Masculin/Féminin, La pensée de la différence, Paris, Odile Jacob 1996 et Masculin-Féminin 2, Dissoudre la biérarchie, Paris, Odile Jacob 2002, cité désormais MasculinFéminin 2.

Masculin-Féminin 2 (supra, n. 6), p. 20 : «L'importance et la quasi-universalité de ces représentations qui dessaisissent les femmes de leur capacité brute de fécondité montraient assez que le moteur de la hiérarchie était là : dans l'appropriation de la fécondité et sa répartition entre les hommes.

${ }^{8}$ Masculin-Féminin 2 (supra, n. 6), p. 23.

9 Masculin-Féminin 2 (supra, n. 6), p. 21.
} 
Héritier c'est, non pas pour m'en prendre à son travail qui est à bien des égards admirable, mais à cause de l'impact éditorial de thèses qui ont donné à certaines observations de Nicole Loraux, convoquée à l'occasion, une formidable caisse de résonance. Ce sont donc moins sur les raisons invoquées par l'anthropologue pour expliquer le phénomène de la domination masculine que je vais m'arrêter, que sur les arguments de Nicole Loraux, essentiellement à partir de son étude du Ménexène de Platon, que celle-là utilise et diffuse.

\section{Les propositions de Nicole Loraux}

Au centre des études de l'helléniste, nous trouvons une affirmation essentielle, celle qui lui fait considérer la terre mère, entendue comme représentation mythique, comme une figure particulièrement efficace politiquement. Cette efficacité fonctionne sur trois niveaux que je rappelle ici pour souligner l'enjeu de la démonstration de Nicole Loraux ${ }^{10}$.

Cette figure mythique permet d'abord de naturaliser la démocratie : « Nous

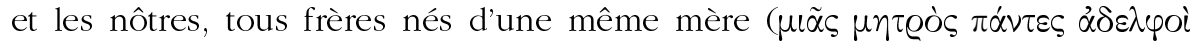

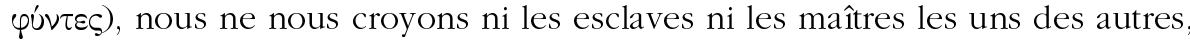

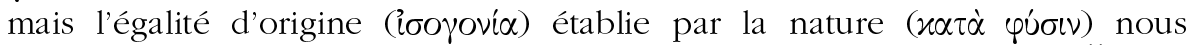

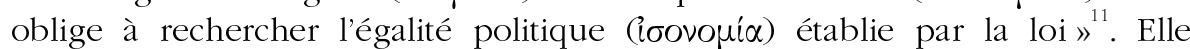
permet ensuite d'anoblir le genos athénien : l'isogonia est eugeneia. Ce faisant elle permet d'enraciner ce régime dans les représentations aristocratiques

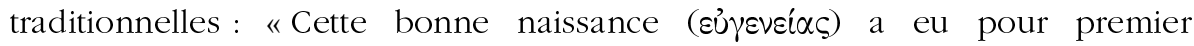
fondement l'origine de nos ancêtres qui, au lieu d'être des immigrés et de faire de leurs descendants des métèques dans le pays où ils seraient euxmêmes venus du dehors, étaient des autochtones, habitant et vivant vraiment

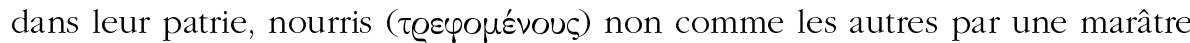
mais par la terre, leur mère (érò $\mu \eta \tau \varrho o ̀ \varsigma \tau \tilde{\eta} \subseteq \chi \dot{\omega} \varrho \alpha \varsigma){ }^{12}$. Enfin, et nous trouvons ici une des sources de l'argumentation de Françoise Héritier, cette figure mythique permet de déposséder les femmes de leur rôle dans la reproduction sexuée. Ainsi, à propos de Pandora et de ce genos pensé séparé de celui des hommes, en particulier chez Hésiode, elle commente : «Certes, il faut, dans la

10 Dans la bibliographie considérable que Nicole Loraux a consacrée à cette question, citons "'Naître'. Origines des hommes. Naître enfin mortels », in Y. BONNEFOY (éd), Dictionnaire des mythologies II, Paris 1981, p. 197-202; ead., Les Enfants d'Athéna. Idées athéniennes sur la citoyenneté et la division des sexes, Paris, 1981, notamment p. 44 et 66; ead., "Les bénéfices de l'autochtonie », Le Genre humain 3 / 4 (1982), p. 238-253; ead., "Débouter le féminin ou la ruse d'un mythe », Psychanalystes 13 (1984), p. 3-16; ead., "Variation grecque sur l'origine. Gloire du Même, prestige de l'Autre » Cahiers de l'école des sciences philosophiques et religieuses 2 (1987), p. 69-94; ead., "La terre, la femme. Figures anciennes, constructions modernes », Peuples méditerranéens 56-57 (1991), p. 7-17; ead., "Pourquoi les mères grecques imitent, à ce qu'on dit, la terre ", Nouvelle revue de psychanalyse 45 (1992), p. 161-172. N. LORAuX, Né de la terre. Mythe et politique à Athènes, Paris, 1996, reprend ces différents articles que je citerai désormais dans cette édition.

11 Ménexène, 238e-239a, cité ainsi dans Né de la terre, o.c. (n. 10), p. 41.

12 Ménexène, 237b, cité dans Né de la terre, o.c. (n. 10), p. 39 
réalité, qu'elles enfantent des fils semblables à leur père : telle est même en toute cité la définition du bon ordre social. Mais, au niveau de la pensée mythique, les hommes grecs, avec un frisson de plaisir terrifié, préfèrent enfermer les femmes dans un genos toujours prêt à faire sécession, voire à se reproduire en circuit fermé : opération fructueuse de l'imaginaire masculin, qui libère le champ pour le fantasme inverse - le vrai, n'en doutons pas d'une reproduction qui, enfin, n'aurait pas besoin de passer par les femmes $»^{13}$. Et on connait sa conclusion, donnée à propos de l'oraison funèbre de Démosthène $^{14}:$ : Il me suffira de constater ici que la Terre-Mère débarrasse à point nommé les Athéniens de l'autre sexe et de sa fonction reproductrice ${ }^{15}$. La cité est totalement celle des pères : il n'y a plus de place pour les femmes $^{16}$. On le constate, il est non seulement question ici de rivalité entre la terre et les femmes (ce qui est une lecture du Ménexène sur laquelle nous reviendrons) mais également de rivalité entre les femmes et les hommes puisque ce qui est en jeu est leur capacité reproductive respective. D'ailleurs, au préalable, Nicole Loraux évoque, à propos des hommes, «leur fascination du féminin et leur peur des femmes ${ }^{17}$.

Bien sûr, on relèvera, après Nicole Loraux, l'ambiguité de cette figure de la terre mère dans laquelle il faut distinguer la terre mère de l'humanité (celle que, comme la Gaia hésiodique ou la glèbe qui porte les fruits, Platon associe sournoisement dans son oraison funèbre parodique au territoire de la cité) et celle, civique, mère des seuls citoyens (celle de l'oraison funèbre traditionnelle, non subvertie). Quoi qu'il en soit, ce motif, même contextualisé dans son environnement militaro-civique, repose sur une pensée de la maternité de la terre qui est plus générale et sur une représentation bien masculine du féminin qu'il faut préciser. Avant d'en venir là, précisons en quoi la notion de genre permet de renouveler ces questions.

\section{La notion de genre}

Ce que l'on exprime par la notion de genre est l'idée selon laquelle l'identité sexuée est très largement constituée par des faits culturels. Dans cette perspective, l'historien est amené à mettre sérieusement en doute les déterminismes biologiques souvent convoqués pour expliquer les assignations

\footnotetext{
13 Né de la terre, o.c. (n. 10), p. 43.

14 Démosthène, Epitaphios 4 : «Ce n'est pas seulement à un père que l'on peut, pour eux et pour chacun de leurs lointains ancêtres, faire remonter individuellement leur naissance ( $\tau \dot{\eta} v$

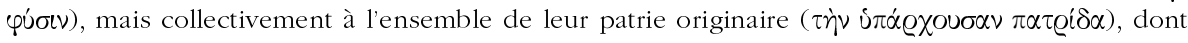

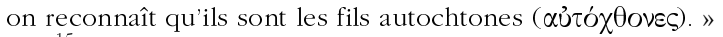

15 Né de la terre, o.c. (n. 10), p. 44.

16 Néanmoins ce processus ne s'effectue pas sans révéler en même temps cette place essentielle que les hommes réservent aux femmes : "Dans un cas comme dans l'autre et que ce soit pour en doter une terre primordiale ou une terre déjà passive et fécondée par un semeur, ce qu'il convient d'ôter aux mères pour les évincer de l'origine, c'est la grossesse et la reproduction, dont pourtant les hommes grecs leur font à la fois une nature et le plus civique des devoirs » (Né de la terre, o.c. [n. 10], p. 138).

${ }^{17}$ Né de la terre, o.c. (n. 10), p. 79.
} 
selon le sexe. Il est appelé à une nouvelle vigilance en ce qui concerne l'analyse des rapports hommes/femmes et leurs représentations. Or ces deux niveaux d'analyse ne se recouvrent pas exactement dans la mesure où les genres ne coïncident pas nécessairement avec les hommes et les femmes comme individus sexuellement déterminés.

Davantage encore, ce regard donne une dimension historique à la différence des sexes. Autrement dit la césure qui nous semble si fondamentale entre hommes et femmes est également considérée comme une construction sociale : c'est la constitution des genres, masculin et féminin, qui a conduit à penser les corps comme radicalement différents et qui a inscrit cette différence dans la sexualité de la reproduction. La réalité de la différence sexuelle a été ainsi considérablement renforcée et durcie parce que c'est elle qui fut censée représenter la différence des genres. Il semble que dès l'Antiquité les philosophes aient introduit une telle lecture des corps et du biologique même si la diversité des opinions ne laissait pas émerger une seule théorie normative comme ce sera le cas à partir du XVIII ${ }^{\mathrm{e}}$ siècle $^{18}$.

Ces remarques conduisent à nous méfier de notre propre tendance à lire les sources antiques en y projetant le schéma de la bicatégorisation des sexes/genres, celle d'une opposition qui serait fondamentale car biologiquement déterminée. Par rapport à notre dossier, ce regard critique conduit à douter de l'hypothèse d'une puissance féminine maternelle originelle et autonome, substrat «invariant » ${ }^{19}$, qui ferait, par définition, peur aux hommes, autre «invariant», mais d'ordre psychologique cette fois. Elle conduit à s'intéresser davantage aux fonctions mises en jeu dans ces questions plutôt qu'aux personnes. Elle invite à poser quelques questions simples: Les hommes grecs ont-ils pensé la maternité (qui constitue seulement un aspect du féminin) comme une puissance? L'ont-ils pensée comme une puissance intolérable? Et si l'on refuse l'argument de la récupération par les hommes du pouvoir des mères, comment comprendre la place si importante de la maternité de la terre dans la rhétorique politique?

${ }^{18}$ T. LAQUeUR, La fabrique du sexe, Essai sur le corps et le genre en Occident, Paris, 1992 [or. angl. 1990]; A. JAulin, « La fabrique du sexe, Thomas Laqueur et Aristote », Clio, Histoire, Femmes et Sociétés 14 (2001), p. 195-205, avec la réponse de T. Laqueur in Clio 15 (2002), p. 209-211; H. KING, Hippocrate's woman. Reading the female body in Ancient Greece, Londres / New York, 1998; C. KRAUS, "La bicatégorisation par sexe à l'épreuve de la science' ", in D. GARDEY, I. LÖwY (dir.), L'Invention du naturel. Les sciences et la fabrication du masculin et du féminin, Paris, 2000, p. 187-213; en dernier lieu, J.-B. BONnARD, Le Complexe de Zeus, Représentations de la paternité en Grèce ancienne, Paris, 2004, désormais cité J.-B. Bonnard, Le complexe de Zeus.

${ }^{19}$ Le terme est choisi à dessein et renvoie aux débats autour du premier Masculin-Féminin de Françoise Héritier qui évoquait la "valeur différentielle des sexes» comme un invariant. Michèle Riot-Sarcey me rappelait que sur ce point la position de l'anthropologue avait sensiblement évolué puisque dans le second volume elle lui substitue le terme de «matrice». Sur ces débats voir en particulier "Autour du livre de Françoise Héritier Masculin-Féminin: Dissoudre la Hiérarchie », dans la rubrique "Controverse » in Travail, Genre et Société 10 (2003), p. 173-217, avec les contributions d'Agnès Fine, Pascale Molinier, Sabine Prokhoris, Alice Pechriggl, Marie-Blanche Tahon, et la réponse de Françoise Héritier. 


\section{Produire la vie, un acte à la fois féminin et masculin}

Il faut rappeler ce constat, qui émane en particulier de la Théogonie d'Hésiode, la pensée grecque n'a pas mis la terre, ou la mère, ni le père seul, à l'origine de la vie. Chez Hésiode, il existe bien une génération spontanée qui débute avec Chaos, "qui naquit aux tout premiers temps ( $\pi \varrho \tilde{\omega} \tau \iota \sigma \tau \alpha)$ »,

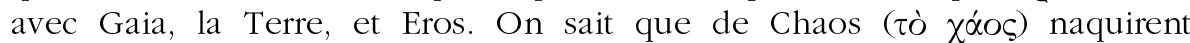

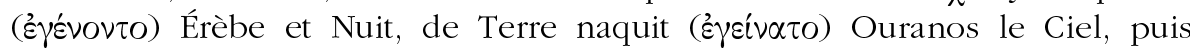
Ouréa les Hauts Monts, Pontos l'espace marin, avant que Gaia, la Terre, n'enfante ensuite la génération des Cronides $^{20}$. Dans ce processus générationnel, avant que la mixité ne s'impose dans la Théogonie avec Gaia et Ouranos, les puissances fécondantes sont sexuellement indifférenciées, ce qui signifie que les fonctions maternelle et paternelle, qui seront ensuite dissociées, peuvent alors être pensées au sein d'une même entité divine, qu'elle se présente au féminin, au masculin ou au neutre ${ }^{21}$. Ceci ne veut pas dire que père et mère soient mis sur le même plan, car la désignation du père dans la Théogonie, associée à une culture très patrilinéaire, comporte un enjeu social, la souveraineté, que ne revêt pas celle de la mère ${ }^{22}$.

Avoir souligné que la production de la vie est pensée avec les deux genres, que le primat du père relève d'une culture où la transmission des statuts et des biens passent par l'homme, n'a pas pour corollaire une négation du principe fécondant de la mère à laquelle est souvent associée la terre. Il existe toute une tradition poétique pour développer ce thème. Ainsi la terre fertile est qualifiée de mêtêr, de pammêtor ou exceptionnellement, pour Colone en Attique, de matripolis ${ }^{23}$. Mais dans ces occasions est également mis en avant le rôle du fleuve qui apparait alors comme un principe masculin. Dans le bourg de Colone évoqué par Sophocle les vallons sont verdoyants, signe d'une terre, épaisse et riche, parce que fertilisée par le Céphise. Son "flot vagabond»,

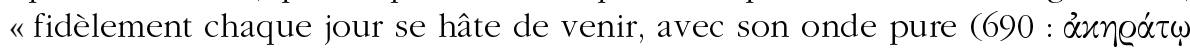

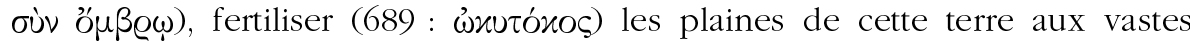

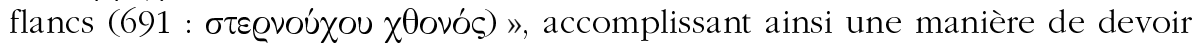
conjugal métaphorisé dans la tradition grecque par l'image de la femmechamp et du sexe-sillon ${ }^{24}$.

\footnotetext{
${ }^{20}$ Hésiode, Théogonie, 45, 123, 126 sq.

${ }^{21}$ T. COLE, Democritus and the sources of Greek anthropology, Cleveland, 1967, p. 22 sq. et 174-192; L. BRISSON, Le sexe incertain. Androgynie et hermaphrodisme dans l'Antiquité grécoromaine, Paris, 1997 , p. 130

${ }^{22}$ Il est intéressant de noter que le terme de mêtêr n'est pas utilisé dans le chant des Muses de la Théogonie (26-74), au contraire de pater, pour Zeus (Théogonie, 37, 40, 47, 53, 71) et une seule fois pour Cronos désigné comme père de Zeus (Théogonie, 73). Dans l'Iliade le couple originaire est formé d'Océan et de Téthys (XIV, 201).

23 Pindare, Néméennes VI, 1 sq.; Eschyle, Sept contre Thèbes, 584; Eschyle, Prométhée, 90; Sophocle, Edipe à Colone, 707-708 (voir aussi Sophocle, Philoctète, 391 sq.). Sur cette association de la terre et de la mère, P. Chantraine, "Les noms du mari et de la femme, du père et de la mère en grec », REG 59-60 (1946-1947), p. 219-250, p. 239.

24 J.-B. BONNARD, Le complexe de Zeus, o.c. (n. 18), p. 105-115.
} 
Nicole Loraux, en évoquant la naissance des êtres dits «primordiaux », ces ancêtres des hommes qui font la transition entre l'origine et le temps des hommes, rappelle qu'ils sont issus de deux éléments de la nature dont un fleuve : Phoroneus a ainsi pour parents le fleuve Inachos et la nymphe Melia, elle-même fille de l'Océan ou soeur des Meliai, les nymphes des frênes nées de Gaia fécondée par le sang d'Ouranos ${ }^{25}$. Selon ce schéma classique, de l'association du fleuve et de la terre naissent des plantes mais aussi parfois des hommes, voire des villes, ainsi Égine ou Thèbes chez Hérodote ${ }^{26}$.

La terre comme mère apparaît ainsi très rarement seule dans le processus de génération et la notation du Ménexène selon laquelle la terre athénienne a enfanté (eteken) les ancêtres des Athéniens, seule cette fois puisqu'elle a à la fois porté et engendré (kuein et gennaein), doit, de ce point de vue, être soigneusement isolée ${ }^{27}$. De façon plus générale un principe masculin et un principe féminin sont à l'origine de la vie.

Par ailleurs la frontière entre reproduction de la nature et reproduction humaine est particulièrement difficile à tracer. Nicole Loraux a pensé pouvoir isoler des verbes caractérisant chacun de ces domaines : phuein serait le verbe de la reproduction générale et tiktô / teknoô celui de la reproduction humaine. Cette distinction constitue son argument majeur pour dire que le Ménexène, en attribuant le tiktein à la terre (237e) usurpe la fonction reproductive des mères, les femmes ${ }^{28}$. C'est ainsi la terre, qui «imite les femmes», et non l'inverse comme veut le faire croire Platon dans le Ménexène!

Ce choix décisif de Nicole Loraux a en réalité occulté la diversité des emplois du Ménexène pour désigner l'acte par lequel la terre a donné la vie aux premiers Athéniens. La flexibilité lexicale est en effet beaucoup plus importante : phuein, tiktein, kuein, gennaein, trophên echein, sont des verbes employés pour renvoyer à cette action «originelle» de la terre de l'Attique dans le texte platonicien ${ }^{29}$.

Mais la question que soulève Nicole Loraux n'est pas seulement celle d'un partage entre puissance de la nature et reproduction humaine, il s'agit aussi de ranger la terre du côté des femmes et donc de procéder à une répartition des fonctions entre les hommes et les femmes. Les choses paraissent assez

\footnotetext{
25 N. LORAuX, Né de la terre, o.c. (n. 10), p. 12-13.

${ }^{26}$ Hérodote, V, 80; Euripide, Phéniciennes, 666-669.

27 Platon, Ménexène, 237e. On notera au passage que ce crédit de puissance est en même temps, dans l'argumentation du Ménexène, reconnu aux femmes. L'bymne homérique à la Terre est parfois invoqué pour étayer l'ancienneté et la notoriété de la représentation de la terre comme mère. La Terre y est certes celle qui fournit la vie (bios) mais elle est également épouse (alochos) d'Ouranos dont elle partage donc la couche. Par ailleurs sa prééminence, dans ce texte, peut être mise en relation avec le genre même de l'hymne, une "invocation » à l'adresse d'une divinité particulière, comme me le rappelait Stella Georgoudi.

${ }^{28} \mathrm{La}$ formule du Ménexène (237e: pan to tekon) qui "généralise et désexualise l'enfantement » (Né de la terre, p. 130-131) reprend une expression de l'Ion d'Euripide (542: ou pèdon tiktei tékna).

${ }^{29}$ Ménexène, 237a, 237c, 237d, 237e, 238a, 239a
} 
évidente si l'on se range à la déclaration d'Oreste, jugé pour avoir tué sa mère puis acquitté parce qu'il vengeait ainsi le meurtre de son père : «Si je suis un impie d'avoir tué ma mère, je porte un autre nom, celui d'homme pieux, car j'ai vengé mon père. Quel était mon devoir? À deux raisons, opposes-en

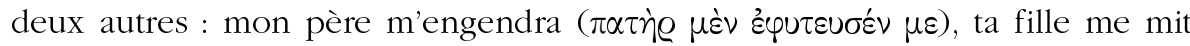

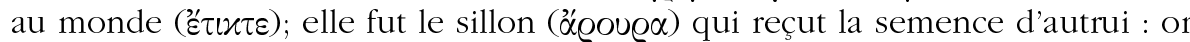

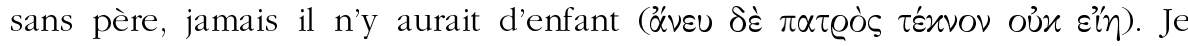
pense donc que l'auteur de mes jours ( $\tau \tilde{\varphi}$ jévous $\alpha \varrho \chi \eta \gamma \varepsilon ́ \tau \eta)$ avait droit à mon

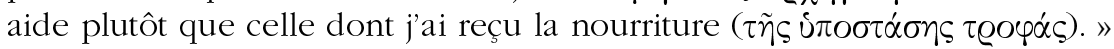

Le héros d'Euripide développe ici l'argumentation bien connue de l'Apollon d'Eschyle dans les Euménides ${ }^{30}$. Celle-ci s'appuie sur une tradition ancienne - que Jean-Baptiste Bonnard a récemment éclairée - que l'on peut faire remonter à Anaxagore et qui va devenir très influente par l'autorité d'Aristote. Selon le Clazoménien, «la semence en effet est engendrée par le

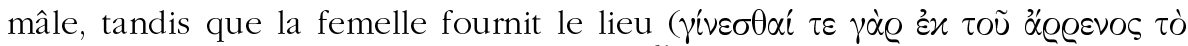

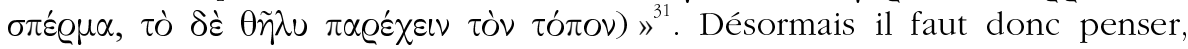

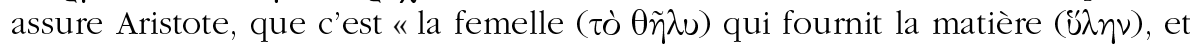

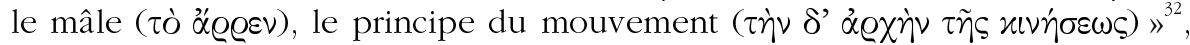

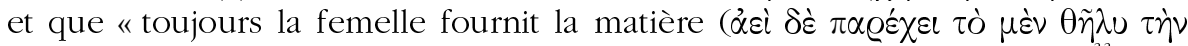

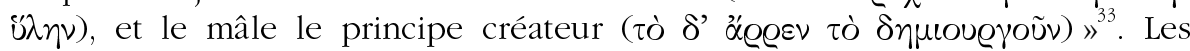
partages en termes de semence, de plantation (phuteuein, phuein) et de délivrance, accouchement (tiktein) semblent alors acquis. Surtout, la hiérarchie des genres à laquelle ces partages conduisent, point sur lequel JeanBaptiste Bonnard insiste à juste titre, s'inscrit désormais dans les corps et en particulier dans le processus de reproduction.

Pourtant, et sans même tenir compte du dossier si difficile à interpréter des Présocratiques et des Hippocratiques, la tradition littéraire antérieure à Aristote effectue des partages beaucoup moins simples : dans l'Iliade, le père enfante (tiktô) aussi, comme Ouranos dans la Théogonie ${ }^{34}$. À l'inverse, dans l'Ion d'Euripide si Xouthos «engendre » son fils avec le verbe $p h u \hat{o}^{35}$, ce qui semble conforme, sinon à la vérité du personnage, du moins à la norme des genres, Ion emploie le même verbe pour évoquer sa mère ( $\tau^{\prime} v o s$ dé oo $\pi \varepsilon ́ \varphi u \varkappa \alpha \mu \eta \tau \varrho o ́ \varsigma ;)^{36}$. Les traducteurs en revanche préfèrent, à défaut semble-t-il de pouvoir penser l'engendrement maternel, la périphrase «qui est ma mère? ${ }^{37}$ !

30 Euripide, Oreste, 546-556; Eschyle, Euménides, 657-673.

31 Anaxagore, fr A 107 = Aristote, Génération des Animaux (désormais G.A.) IV, I, 763b 30.

32 Aristote, G.A. II, 4, 740b 24-25.

33 Aristote, G.A. II, 4, 738b 20-24

34 Iliade II, 628; VI, 206, etc. Hésiode, Théogonie, 43-45; 208. Même Aristote n'est pas toujours si tranché dans les partages des fonctions reproductives en fonction des genres : G.A. I, 18, 724b 14-15, cité par J.-B. Bonnard, Le Complexe de Zeus, o.c. (n. 18), p. 175.

Ion, 536-537.

36 Ion, 540.

37 Traduction de Léon Parmentier et Henri Grégoire, édition des Belles Lettres. 
Ces constatations, qui ne valent qu'à titre d'exemples, nous engagent à nous méfier d'une projection sur les textes classiques d'une conception somme toute très aristotélicienne, et sans doute satisfaisante pour un esprit rationnel, d'une représentation très hiérarchisée de la procréation qui assigne à la femme le seul rôle de la fameuse "cause matérielle " ${ }^{38}$. Si l'opposition engendrement / enfantement n'est pas une césure fondamentalement significative d'un partage masculin/féminin dans la tradition littéraire archaïque ou classique, une autre fonction a pu apparaitre davantage pertinente pour spécifier les genres des tâches reproductives, c'est celle de la trophê.

Cette fonction est particulièrement soulignée par Stella Georgoudi à propos du Ménexène : «En résumant, je dirais que, dans le Ménexène, la terre ne se présente pas tellement comme la mère originelle, comme une toutepuissante reproductrice' ou comme un champ auquel la femme doit s'identifier, 'dans sa fonction procréatrice', un champ qui a besoin d'un arotêr, d'un 'laboureur'. Dans ce texte, la terre apparaît surtout comme le modèle nourricier que la femme a imité et continue d'imiter, dans la grossesse et l'enfantement. Tout se joue donc autour de la production de la trophêe, ce qui expliquerait sans doute l'absence, dans ce passage de 'tout principe masculin' " ${ }^{39}$. Ce déplacement de la question de la procréation à celle de la fonction nourricière me paraît effectivement fondamental car bien souvent repéré dans les sources (l'Oreste d'Euripide cité plus haut oppose le père archégète et la mère nourricière). Si je ne souscris pas à la conclusion de Stella Georgoudi sur le partage des genres qu'opère la trophế $\hat{e}^{40}$, je retiendrais en revanche l'importance de cette notion pour dire la fabrication de la vie, surtout parce qu'elle est d'un intérêt majeur dans le contexte de la rhétorique patriotique qui place au centre de sa logique celle de la réciprocité des bienfaits ${ }^{41}$. Or justement les pièces du dossier, rassemblées par Nicole Loraux pour étayer l'hypothèse de la dépossession de la maternité des femmes au profit de la terre, renvoient à ce contexte particulier dont on ne prend souvent pas suffisamment en compte la nature spécifique.

38 Aristote, G.A. II, 1, 732 a 6-9 : J.-B. BONNARD, Le Complexe de Zeus, o.c. (n. 18), p. 186.

39 S. GEORGOUd, «Gaia/Gê. Entre mythe, culte et idéologie », l.c. (n. 2), p. 132-133.

40 Dans la mesure où la trophê est très souvent paternelle et pas seulement dans le domaine de l'éducation, de la «nourriture sociale». Dans les partages terre/fleuve le fleuve nourrit, tout autant que la terre (Sophocle Oedipe à Colone, 673, 687-693; L. ROBERT, «Documents d'Asie Mineure », BCH 101 [1977], p. 130). Sur la trophê masculine voir le dossier de B. STRAuss, Fathers and sons in Athens, Londres, 1993. Dans la Cité au banquet (Paris / Rome, 1992, p. 212-213, et à propos d'Ion, p. $472-490$ et p. 530 sq.) Pauline Schmitt Pantel a largement démontré combien la trophê circulait entre le dieu, la pythie, et la mère Créuse (qui a été en défaut de trophê) et se montrait ainsi indifférente aux genres. On ne saurait alors assimiler l'aspect nourricier de la terre du Ménexène a une fonction spécifiquement féminine.

41 Sur cette notion de réciprocité, C. GILL, N. POSTLETHWAITE, R. SEAFORD, Reciprocity in Ancient Greece, Oxford, 1998, p. 279-301, et V. Azoulay, Xénophon et les grâces du pouvoir, Paris, 2004. 


\section{Terre maternelle et rhétorique patriotique}

En effet, le corpus, mis à part les notations littéraires et poétiques évoquées plus haut, est essentiellement constitué autour des récits sur l'autochtonie athénienne, notamment on l'a compris le Ménexène parodique, et dans une moindre mesure thébaine.

Le motif de la terre mère est alors une figure qui intervient relativement tardivement. Il est absent de l'Iliade et des vers de Tyrtée dans lesquels on meurt certes pour la terre (gê) mais une terre qui n'est jamais désignée comme mère. Il en est de même chez Théognis où l'on rencontre une patris, une patrê voire une patrôa gê, sans qu'il soit question de terre mère. Chez Pindare, la patra, patrê, ou patris, n'est pas non plus liée à la terre mère et renvoie bien plus explicitement à la notion d'héritage des pères ${ }^{42}$. Dans le péan de Salamine, il s'agit des enfants des Grecs, des sanctuaires des dieux

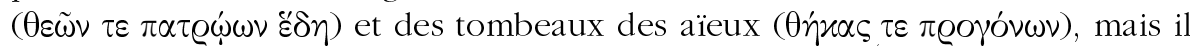
n'y a toujours pas de référence à la maternité de la terre ${ }^{43}$. La terre y est terre des pères (patris), terre de la liberté (eleutheria), jamais terre mère. Chez Thucydide, la cité de l'oraison funèbre est cité nourricière certes mais, comme Nicole Loraux l'a bien montré, sans référence à l'idée d'autochtonie ou alors faut-il comprendre celle-ci au sens restreint de l'indigénat des Athéniens (ils habitent là depuis "toujours »"). La valeur des citoyens, exaltée par Périclès, repose sur leurs seuls exploits, leurs actes, ceux-là seuls qui produisent la puissance (dunamis) de la cité ${ }^{-45}$.

En revanche, dans les Sept contre Thèbes, représentés en 467, il s'agit, dit le prince thébain Etéocle à ses concitoyens, de "porter secours ( $\alpha \varrho \emptyset \eta ́ \gamma \varepsilon \iota \nu)$ ) à la

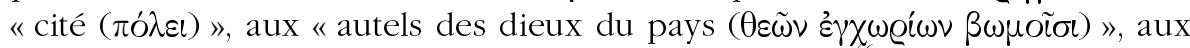

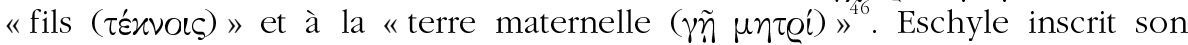
héros Étéocle dans la logique de la guerre pour la patrie, telle qu'elle a été définie par l'Hector de l'Iliade et par Tyrtée, avec des verbes signalant la défense du territoire, amunô, arêgô, boêtheố ${ }^{47}$ mais en y associant, pour la première fois semble-t-il, le thème de la terre mère.

Le motif est ensuite repris dans les Phéniciennes d'Euripide, dans les fragments de l'Érechthée, chez les orateurs, Lysias, Isocrate, Démosthène et Lycurgue, comme chez Platon qui est le seul à parler de mêtris, terme qu'il donne comme un équivalent crétois de la patris des Grecs ${ }^{48}$. Tous ces textes ont en commun de développer le thème de la logique patriotique, celle de l'attachement des citoyens à leur communauté. C'est donc en analysant sa

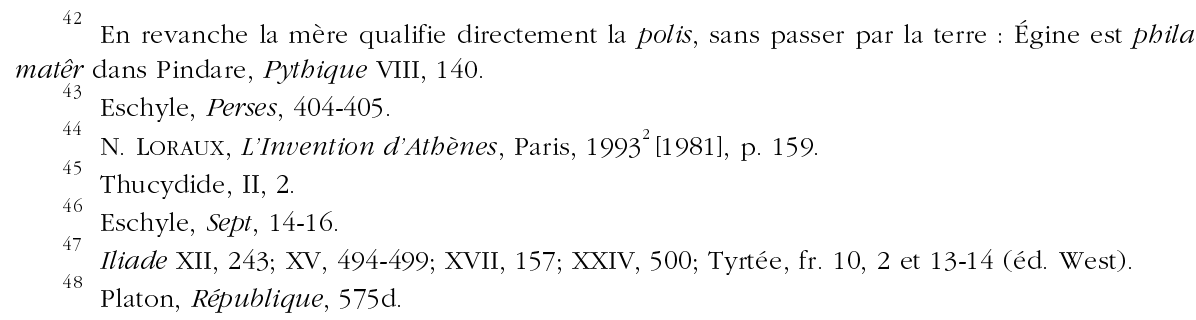


fonction dans cette logique que l'on peut, me semble-t-il, comprendre ce motif.

C'est à dessein, pour rendre à ce corpus large sa cohérence, que j'ai employé l'expression de rhétorique patriotique. En ce qui concerne la notion de rhétorique, je me réfère au sens que lui donnaient Gorgias et Platon pour qualifier un discours qui vise à persuader l'auditeur, l'émouvoir, l'entraîner vers l'action ${ }^{49}$. Je qualifie ce discours de patriotique dans la mesure où l'ensemble du corpus met en avant le renforcement ou l'exaltation du lien à la cité, cette terre civique que les Grecs nomment patris. Ces propos visent à susciter un comportement de dévouement, y compris jusqu'à la mort pour la cité et ne correspondent à aucun genre littéraire. À vrai dire ils traversent presque tous les genres canoniquement répertoriés ${ }^{50}$, souvent sous la seule forme d'allusions, de syntagmes, d'apartés, dans le reste d'un discours qui n'est pas aussi performatif ${ }^{1}$.

Or, lorsqu'il s'agit de toucher l'auditeur ${ }^{52}$, ce qui importe ce n'est pas la capacité de produire des êtres, la puissance en cause dans un rapport de force, qu'il s'institue entre la terre et les femmes ou entre les terre et ses rejetons humains, mais le lien créé par cette naissance autochtone.

En analysant les Sept contre Thèbes, H.D. Cameron a insisté sur le rôle de la gêrotrophia, repris à propos de chacun des Spartes, et bien sûr parmi eux le Ménécée plus longuement décrit des Phéniciennes, mais son argumentation est passée assez inaperçue ${ }^{53}$. Pourtant elle me semble absolument caractéristique du lien patriotique qui se développe ensuite de façon très politique et normative chez les orateurs athéniens du IV siècle. Avant les Sept on pourrait dire que ce lien reposait davantage sur l'identification à la terre habitée ou sur un statut qui passait par la possession d'une certaine terre. Après les Sept le rapport introduit par l'idée de la naissance de la terre même que l'on habite devient central. Il engage une réciprocité contraignante car hiérarchisée et fait du territoire l'enjeu central du combat ${ }^{54}$.

49 Platon, Gorgias, 453a; Gorgias, Éloge d'Hélène, 14, [éd. M. TASinATO, 1990] et L. Pernot, La Rhétorique dans l'Antiquité, Paris, 2000, p. 33. Cette rhétorique est radicalement différente de celle que décrit Aristote en ce que celui-ci ajoute le logos (construit à l'aide de preuves) au pathos éprouvé par l'auditeur et à l'ethos de l'orateur (Rhétorique, 1356a 14). Sur cette forme de rhétorique et ses liens avec la pratique historienne, voir C. GINZBURG, Rapports de force. Histoire, rhétorique, preuve, Paris, 2003 [or. ital. 2000].

50 Aristote, Rhétorique I, 1355b 25.

51 Voir en particulier Thucydide, VII, 69, qui rapporte un discours patriotique (une harangue de chef) qui n'est pas sans rappeler le péan de Salamine rapporté par Eschyle, Perses 403-405.

52 Platon, Ménexène, 235a-d. Voir aussi Lycurgue, Contre Léocrate, 107, qui cite les élégies de Tyrtée composées pour inspirer aux Spartiates la résolution de mourir pour la patrie.

53 H.D. CAMERON, «The Dept to Earth in the Seven Against Thebes », TAPhA 95 (1964), p. 18; id., Studies on the Seven against Thebes of Aeschylus, La Haye, 1971.

54 Le mythos de la République de Platon (414d-e) le souligne assez: il s'agit de créer un rapport de dépendance des citoyens à leur territoire dans la logique de l'attachement et plus précisément de la dette. L’argument est repris dans Isocrate, Panathénaïque, 124-125; Démosthène, Epitaphios, 2-5. 
Il est vrai que dans le mythe thébain la violence de la fondation introduit un élément qui est de l'ordre de la compensation. À l'affront fait à la terre, par le meurtre du dragon, les Spartes bénéficiaires de cette mort, et dans une certaine mesure la cité entière de Thèbes, doivent payer. La contrainte évoquée par le Ménécée des Phéniciennes est donc d'une qualité particulière ${ }^{55}$ : il est un " enfant sorti de la mâchoire du dragon ${ }^{56}$ et comme tel destiné à être sacrifié, car comme le formule Tirésias, le sol doit recevoir un fruit en échange du fruit détruit ${ }^{57}$. Mais, parmi les autres Spartes, des Gegeneis, Ménécée est choisi car il est un êitheos, mot rare équivalent masculin des jeunes filles parthenoi qui sont marqués par leur fidélité à leur père. Lui l'est à son genos tant qu'il n'a pas encore contracté alliance, ce qui n'est plus le cas de son frère Hémon, avec un autre lignage ${ }^{58}$. Dans cette logique, la notion de contrainte (anagkê) s'enrichit d'une autre idée, celle de "pureté " montré ailleurs combien l'obéissance structurelle des parthenoi en faisait d'excellentes victimes mythiques (paradigmatiques), modèles pour tous les citoyens, les seuls vrais guerriers à qui la cité demande d'être prêts à mourir sur le champ de bataille ${ }^{60}$.

Ainsi les héros proches des hommes violents de l'âge du Bronze hésiodique, hommes d'Arès comme les Spartes thébains qui de ce fait ont un compte particulier à régler avec la terre dont ils sont issus, ou les citoyens héritiers de l'autochtonie d'Érechthée à Athènes, sont également liés à la terre d'où ils sont sortis et à laquelle ils doivent la vie. Dans ces mythes la terre est métaphore de la mère individuelle. Le père est souvent représenté par un dieu, Arès pour les Thébains, Héphaïstos (voire Zeus derrière Athéna) pour les Athéniens. Pour tous les citoyens adultes, non parthenoi ni Spartes, pour tous ceux qui sont confrontés à la réalité du champ de bataille et à l'exigence de s'y présenter en risquant à chaque fois leur vie, il s'agit de reconstruire cette nécessité comme le rappelle le mythe platonicien de la République $e^{61}$.

Que le terme anagkaios désigne à la fois la contrainte de la nécessité ${ }^{62}$ et le parent par le sang me semble être un indice pour comprendre le poids des figures de la mère et du père, et ici il s'agit bien des deux figures car toutes deux nécessaires pour l'acte biologique de la naissance, ainsi que nous l'avons dit plus haut, dans la construction du lien réciproque contraignant. Autrement dit, dans la logique de réciprocité des bienfaits, les parents, père et

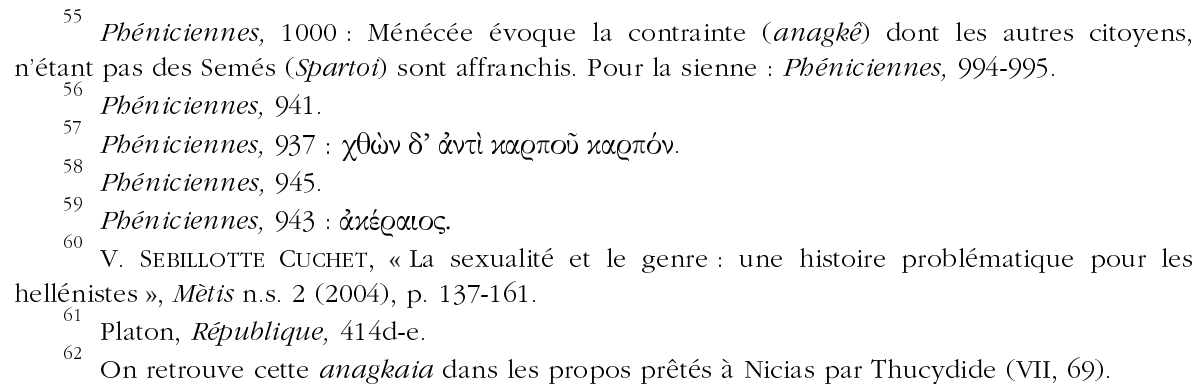


mère sont les créditeurs pour toujours ${ }^{63}$. Ce sont eux qui ont été là avant nous, eux qui sont les palaioi et les archaiotatoi, dépositaires de l'autorité de l'origine (archê), eux qui nous ont toujours nourris avant de réclamer d'être nourris à leur tour ${ }^{64}$. Lorsque la cité reprend à son compte cette logique du don en retour, d'abord construite autour de la relation parents-enfants, elle se dit patris, terre des pères, plus rarement mêtris. Mais même dans la patris (contraction de $\dot{\eta} \gamma \tilde{\eta}$ et de $\delta \pi \alpha \tau \dot{\eta} \varrho$ ) la terre est présente, c'est la gê des pères. La possibilité de jouer sur un attachement (les pères) ou sur un autre (la mère) permet de privilégier une représentation de la cité plutôt qu'une autre. Incontestablement c'est la représentation territoriale qui est associée à la mère.

Or, si la terre récupère si aisément le genre de la mère, il faut convenir que cela s'accorde avec une représentation plus large de ce genre-là comme

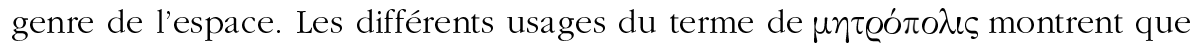
la « maternité » de la cité renvoie d'abord à une notion d'antériorité, comme la "paternité », assez souvent couplée avec une notion hiérarchique, de supériorité, mais signale surtout la localisation d'un espace ${ }^{65}$. Lorsque la métropole est évoquée elle n'est jamais dissociée de ceux qui l'ont construite, les pères ou les ancêtres, et c'est la maternité (mêtropolis) qui traduit l'idée spatiale tandis que la paternité (boi pateres, hoi progonoi) renvoie aux actes, à l'histoire de cette métropole. Le couple pertinent est ainsi constitué de deux fonctions, la maternité associé au singulier du territoire, et la paternité associée à la pluralité des pères et de leurs actions qui construisent l'identité civique ${ }^{66}$. Ce clivage-là me semble un trait culturel relativement stable de la pensée grecque archaïque et classique.

\section{Conclusion : retour sur le Ménexène}

La notion de genre nous a conduite à revenir sur la perspective de la lutte des sexes qui a, ces dernières années, constitué la toile de fond qui semblait éclairer les mythes d'autochtonie. Dans la mesure où non seulement les fonctions sexuées, mais la césure entre le masculin et le féminin, sont comprises comme des constructions culturelles, le premier réflexe est désormais de réfléchir à nos postulats d'analyse. En s'affranchissant autant que faire se peut de nos propres normes de genre, on s'autorise à mieux discerner celles qui se sont mises en place dans les sociétés que nous étudions. Or, dans le cas présent, la norme de genre antique construit une opposition qui me parait moins de l'ordre de la puissance, celle présumée des femmes, et de l'impuis-

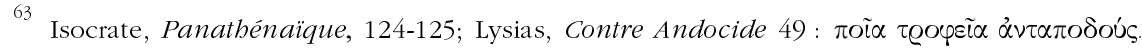
Voir aussi Platon, Lois V, 739e-740a et Lycurgue, Contre Léocrate, 47, 53

${ }_{64}$ Platon, République, 574b-c; Lycurgue, Contre Léocrate, 94.

65 En particulier Platon, Critias, 115c :

66 Hérodote, II, 29, 30; VIII, 31; Thucydide, I, 107; III, 92; Xénophon, Anabase V, 2, 3; 4, 15; 4, 25; Timée, 48e; 50d; 52b; A. Pechriggl, Corps transfigurés. Stratifications de l'imaginaire des sexes/genres, Paris, 2000.
} 
sance, celle présumée des hommes, que de l'espace et des actes, du singulier et du pluriel.

Pour répondre à la question précise de l'autonomie et de la solitude de la terre du Ménexène dans ses enfantements, je proposerais de considérer, non pas un choix personnel de Platon qui serait plus enclin à favoriser la mère, mais la logique du dialogue. Nicole Loraux a insisté à juste titre sur le fait que le Ménexène était un pastiche d'oraisons funèbres athéniennes et surtout de celle de Périclès. Ce n'est pas un hasard que ce soit Aspasie qui la prononce, si l'on pense à la rouerie du Socrate platonicien ${ }^{67}$. Or que dit l'oraison funèbre de Périclès? Que la cité est constituée des pères seuls, les ancêtres, les progonoi, leurs œuvres, les erga. Jamais dans ce discours n'interviennent ni la terre ni la mère. Je me demande donc si, dans le Ménexène, la survalorisation de la mère, y compris dans son autonomie, ne doit pas se comprendre comme un autre effet de dérision par rapport au tout paternel de l'oraison funèbre rapportée par Thucydide. En ce sens l'Aspasie de Socrate serait à l'opposé du Périclès de Thucydide comme les fruits du serment des éphèbes le sont aux murs et arsenaux qu'évoque Lycurgue ${ }^{68}$. Ces positionnements, pour la terre contre les pères, pour les arsenaux ou pour les fruits du territoire, renvoient sans doute aux débats continus des Athéniens sur l'interprétation de ce qu'est leur cité et des choix politiques qu'ils entendent faire. Au coeur de ces questions il y a bien sûr celle de la liberté : doit-on la penser ancrée dans le territoire ou au contraire l'associer à la puissance de la mobilité $^{69}$ ? Dans la construction de l'attachement à la cité il est possible de privilégier soit l'une de ces représentation soit l'autre, ou au contraire de faire jouer les deux ensemble.

Le motif de la terre mère paraît, avec cette lecture par le genre et l'inclusion dans la logique de la rhétorique patriotique, moins liée à une donnée biologique, naturelle, même fantasmée, qu'à un contexte culturel, celui qui associe le féminin de la maternité à l'espace et le masculin de la paternité aux actes et à leur transmission. À mon sens l'usage de ce motif est également lié à une opération bien politique, au sens large, celui de la constitution, chez les Grecs, du lien à la communauté. Il en est une des modalités. Dans l'Iliade ce lien repose sur une image de soi, un statut social associé à un pouvoir enraciné en terre certes, mais sans qu'il soit nécessaire de penser celle-ci comme une mère; dans le traité hippocratique Airs, Eaux, Lieux, la logique de l'attachement est celle d'une identification géomorphologique partagée par tous ceux qui vivent ensemble. Le motif de la terre mère est bien

67 N. Loraux, L'Invention d'Athènes, Paris, 1981, p. 322, 330 et note 26 p. 475.

68 G. DAUX, «Le serment des éphèbes athéniens », REG 84 (1971), p. 370-383, p. 382-383 avait souligné cette opposition à propos de Lycurgue, Contre Léocrate, 150 : la description de la terre riche est bien le choix politique qui, ici, doit permettre de penser leur lien à la terre comme un lien de gratitude envers celle qui nourrit (trephein) les siens. Mais, appuyée sur l'archê construite tout au long du $\mathrm{v}^{\mathrm{e}}$ siècle, c'est la puissance athénienne qui, désormais, est source de bienfaits et réclame un don en retour.

69 Sur cette thématique je me permets de renvoyer à mon livre, Libérez la patrie. L'attachement politique en Grèce ancienne, déposé chez Belin, Paris. 
différent, il est le signe d'un choix politique, au sens restreint cette fois, toujours discutable et discuté qui, en l'occurrence, valorise l'enracinement dans le territoire habité. En ce sens il est historiquement daté, dans l'Athènes des $V^{e}$ et $I^{e}$ siècles. Ce motif ne justifie ni n'explique la domination des hommes sur les femmes dans la société athénienne, voire dans la société grecque antique dans son ensemble: il ne fait qu'illustrer une politique des genres où le féminin est associé au singulier du lieu et le masculin au pluriel des actes, relation absolument asymétrique et fort peu sympathique il est vrai, mais qui n'a absolument rien à voir avec une répartition «naturelle » des fonctions procréatives.

Violaine SEBILLOTTE CUCHET

Phéacie - Université de Paris 1 Panthéon-Sorbonne

Espace Colbert

2 , rue Vivienne

F - 75002 PARIS 